\title{
Detailed Measurement of Wall Strain with 3D Speckle Tracking in the Aortic Root: A Case of Bionic Support for Clinical Decision Making
}

\author{
Sebastian Vogt ${ }^{1,2} \quad$ Konstantinos Karatolios ${ }^{3}$ Andreas Wittek ${ }^{4}$ Christopher Blasé ${ }^{4}$ \\ Anette Ramaswamy ${ }^{5}$ Nikolas Mirow ${ }^{6}$ Rainer Moosdorf ${ }^{6}$
}

\footnotetext{
${ }^{1}$ Department of Heart Surgery, Philipps University, Marburg, Germany

2 Biochemical Pharmacological Center, Cardiovascular Res Lab, Philipps University, Marburg, Germany

3 Department of Cardiology, Universitätsklinikum Marburg und Gießen $\mathrm{GmbH}$, Marburg, Germany

${ }^{4}$ Institute for Cell Biology and Neuroscience, Goethe University, Frankfurt (Main), Germany

${ }^{5}$ Institute for Pathology, Philipps University, Marburg, Germany

${ }^{6}$ Department of Heart Surgery, Universitätsklinikum Marburg und

Gießen GmbH, Marburg, Germany
}

Thorac Cardiovasc Surg Rep 2016;5:77-80.

\begin{abstract}
Address for correspondence Prof. Dr. Sebastian Vogt, Department of Heart Surgery, Philipps University, Baldinger Str. 1, Marburg 35043, Germany (e-mail: vogts@med.uni-marburg.de).
\end{abstract}

\begin{abstract}
Keywords

- aortic disease

- aneurysm

- biomechanics

- echocardiography
\end{abstract}

\section{Introduction}

The lethal risk for patients by formation of aortic aneurysms is still high. Although atherosclerotic process proceeds, first minor symptoms are silent and suggest both patient and practitioner an "apparent security." Quite often, an "enlarged aortic diameter" is measured as benchmark for cardiosurgical repair. In case of the ascending aorta, an increase in diameter up to $5.5 \mathrm{~cm}$ and for the descending aorta an increase in diameter up to $6 \mathrm{~cm}$ are considered as limits, respectively. ${ }^{1}$ However, a marked heterogeneity in the rigidity of the wall of aortic segments can require earlier resection. In case of enlarged aortic roots, the question remains on one hand the earlier the better or surgery just in time of appearing syndromes, although first sign of pain could mean a sudden significant bleeding and urgent emergency. We developed an algorithm on the basis of finite element analysis for evaluation of ultrasonic three-dimensional (3D) speckle tracking data. ${ }^{2}$ With the help of this special investigation, we have established a method for further risk stratification regarding aortic root rupture. In this report, we give an example for further bionic analysis of the aortic root to give a help for received

September 24, 2015

accepted

December 26, 2015

published online

February 4, 2016
DOI http://dx.doi.org/

10.1055/s-0036-1571815. ISSN 2194-7635. (c) 2016 Georg Thieme Verlag KG
Stuttgart · New York

License terms

((1) $\circledast$ 
clinical decision making and optimal schedule of operation. This application was approved by the ethics committee (registration: AZ 131/12; MR 01.2013) and have therefore been performed in accordance with the ethical standards laid down in the 1964 Declaration of Helsinki and its later amendments. The person gave his informed consent prior to his inclusion in the study. The authors obtained consent to publish the data from the patient.

\section{Case Report}

A 70-year-old patient was admitted to hospital with a suspected diagnosis of coronary heart disease. In coronary angiography, high-grade coronary narrowing was excluded. Evidence of an aneurysm of the ascending aorta was found by computed tomographic (CT) scan, whose inner diameter was $52 \mathrm{~mm}$ (-Fig. 1). Aortic elongation led to a shift of the great vessel to the paravertebral left. The aortic valve was bicuspid (BAV), highly sclerotic and there was aortic regurgitation of higher degree. It had two cusps and one raphe and was potentially tricuspid. Two underdeveloped and one fully developed cusps were of non-equal size with one underand two fully developed commissures (classical type $1 \mathrm{BAV}$ with the subcategory $\mathrm{R}-\mathrm{N}, \mathrm{B}(\mathrm{I}+\mathrm{S})$ according to Sievers and Schmidtke). ${ }^{3}$ Corresponding to these findings, we found an aneurysm of the ascending aortic vessel. The aortic root was

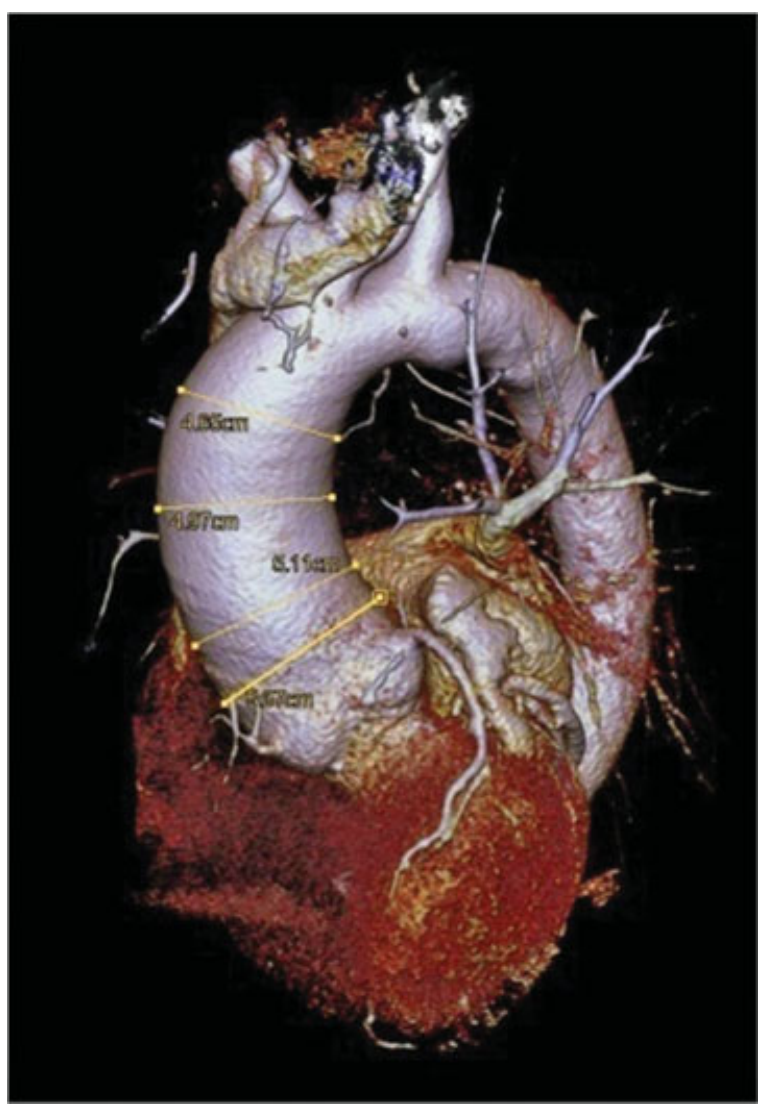

Fig. 1 3D reconstruction of the patients' CT scan. The enlarged aortic root is obvious. Different diameters as typical decision-making tools for surgical intervention at present are seen. not affected. By modified 3D echocardiography of the ascending aorta, specific investigations of the elastic properties of the aortic wall were performed. Aortic imaging was achieved with a commercial real-time 3D-echocardiography system (Artida, Toshiba Medical Systems, Tokyo, Japan) by means of a 3D transthoracic probe (PST-25SX, 1-4 MHz phased array matrix transducer). Aortic wall motion analysis with speckle tracking was performed on the stored 3D raw data by a modified wall motion tracking algorithm with advance cardiac package (Toshiba, UltraExtend, Toshiba Medical Systems GmbH, Neuss, Germany) and circumferential strain (CS) and area strain (AS) were calculated. These data were also further analyzed and processed with a custom-written software and the Abaqus/Viewer 6.10-1 (Simulia, Providence, Rhode Island, United States) to visualize local strains and displacements on 3D models of the aorta. ${ }^{2}$ A grid was placed along each of the intra- and extraluminal interfaces of the aortic segment and those points were tracked. Displacements of the points in the grids allow the documentation of the field vectors which are to be regarded as a measure of the timedependent deformation of the aortic wall. These deformations can draw far more accurate color-coded images in the modeled aortic segment (-Fig. 2a, e). Because of these deformations, longitudinal stain (LS) and CS for the segment were calculated to be 13.6 and $18.1 \%$, respectively (-Fig. 2 ). LS values were not different in concern of normal values $\left(\mathrm{LS}_{\mathrm{N}}\right.$ between 15.2 and $17.1 \%{ }^{4}$ ), but, of course, $\mathrm{CS}_{\mathrm{S}}$ were increased $\left(\mathrm{CS}_{\mathrm{N}}\right.$ between 9.2 and $\left.10.0 \%^{2}\right)$. With the higher diameter of the aortic root (see - Fig. 1), the index of strain heterogeneity $\left(\boldsymbol{I}_{\boldsymbol{H}}\right)$ in this case was lower $\left(0.76\right.$ vs. $\left.1.5 \%^{2}\right)$ and also the systolic dyssynchrony index $\left(\boldsymbol{I}_{\mathbf{d y s}}{ }^{\text {sys }}\right)$ was reduced (14.3 vs. 30.7\% ${ }^{2}$ ), indicating reduced movement of the aortic wall but almost normal rigidity.

AS is a deformation measure that combines LS and CS in one value, thus being a more appropriate deformation measure than CS alone. AS analysis shows no difference to normal data (30.9 vs. $29.9 \%$ ). Distensibility relates the observed deformation (strain) to pulse pressure and describes the elastic properties of the aortic wall. Circumferential distensibility $\left(C D=2.51 \times 10^{-3} \mathrm{~mm} \mathrm{Hg}\right)$ and area distensibility $\left(\mathrm{AD}=4.29 \times 10^{-3} \mathrm{~mm} \mathrm{Hg}\right)$ were determined. $\mathrm{CD}$ is in the range of the values that were observed in healthy volunteers $\left(\mathrm{CD}_{\mathrm{N}}\right.$ between 2.00 and $\left.2.63 \times 10^{-3} \mathrm{~mm} \mathrm{Hg}\right)$. $\mathrm{AD}$ is reduced $\left(A D_{N}\right.$ between 5.98 and $\left.7.86 \mathrm{~mm} \mathrm{Hg}\right)$ indicating increased stiffness of the aortic wall. All the data result in a feature of an enlarged aortic root and subsequent deformations and displacements but rather normal elastic material behavior (-Fig. 2).

\section{Discussion}

The formation of an aortic aneurysm is undoubtedly a dangerous threat. Currently, we expect an age- and genderrelated rupture rate of 3.5 per 100,000 persons. ${ }^{5}$ However, at present in patients older than 65 years, incidence and mortality of ruptured aortic aneurysms was found in less than $2 \%$. This reduction is apparently due to screening programs, which indicates a potential hazard in time. An increase in aortic size beyond a diameter of $5 \mathrm{~cm}$ is associated 

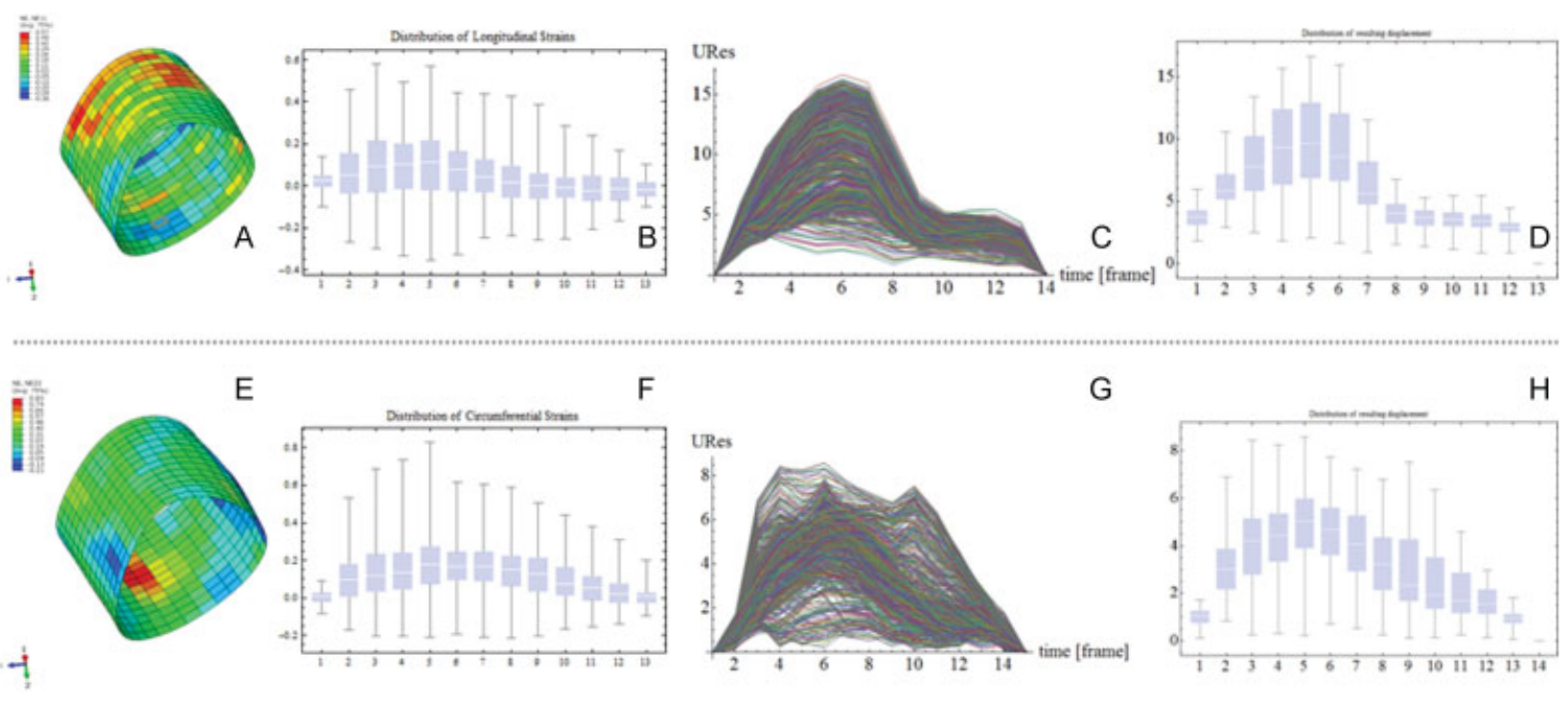

Fig. 2 Results after infinite element analysis of 3D speckle tracking recording of the aortic part of interest. (A) Colored feature of the longitudinal and (B) distribution along the aortic root is shown. (E) Same feature and (F) distribution are seen for the circumferential strain. Final overall resulting displacement time related $(\mathbf{C}, \mathbf{G})$ and its distribution $(\mathbf{D}, \mathbf{H})$ between normal $(\mathbf{C}$ and $\mathbf{D})$ and enlarged $(\mathbf{G}$ and $\mathbf{H})$ aortic roots.

with increased mortality. ${ }^{6}$ Often the experience of the surgeon determines whether aortic root replacement is indicated or not, "even" at $5 \mathrm{~cm}$. With increased diameters, increased aortic wall stiffness was observed. ${ }^{1}$ Beyond such populationaveraged information, an exact description of the mechanical properties of the aortic wall would give a much more accurate tool to identify rupture-endangered aortic segments. Our group uses the high-resolution ultrasound sequences to determine anisotropic elastic properties of the aorta using an iterative finite element updating algorithm. ${ }^{2,4}$

By creating a virtual computer model in silico, a general technical principle will be implemented in a particular medical case. It seems possible in the near future to study biological objects in silico by means of ultrasound and to determine specific characteristics (e.g., elasticity, rigidity, and material coefficients). ${ }^{2}$
In this case according to common indications, the ascending aorta was replaced by prosthesis. According to biomechanics, it remains questionable weather aortic roots in this case have to be replaced. Although the histology represents mucoid degenerations of the aortic wall and lipidosis of the intima (-Fig. 3), the indication remains unclear if in case of severe concomitant diseases (chronic obstructive pulmonary disease, diabetes, progredient arteriosclerosis, stroke, etc.) and higher age, surgical procedure cannot be avoided by successful medical therapy of blood pressure. Therefore, risk stratification appears necessary and nowadays possible of impending aortic rupture. Without any doubt, BAV morphology has an impact on the shape and progression of an aortic type A aneurysm. ${ }^{3}$ The vertical blood flow in the thoracic aorta is changed. ${ }^{7}$ The flow pattern is a major contributor in the pathogenesis of aortic dilation ${ }^{8}$ because

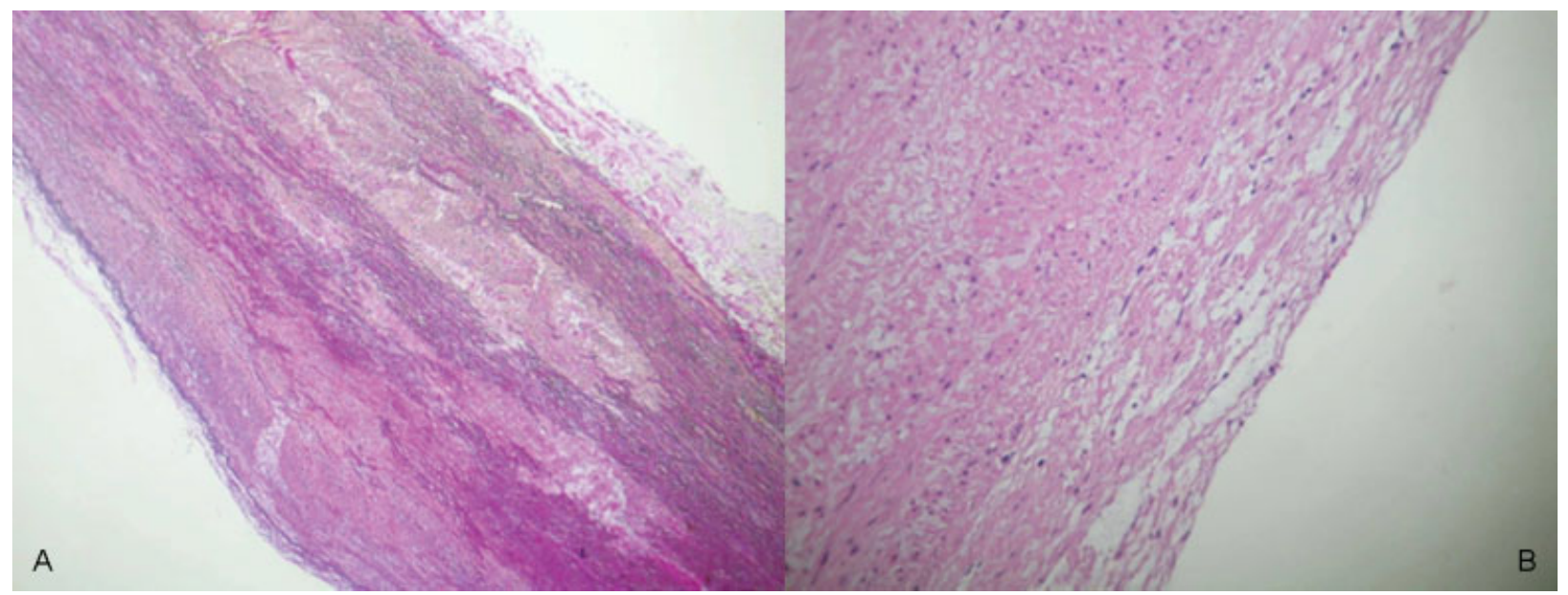

Fig. 3 Histological examination of the patients' aortic wall (EvG staining, 100-fold magnification (A) and HE staining 200-fold magnification (B), respectively). pathological findings results in mucoid degeneration of the aorta (A) with lipidosis of the intimal layer (B). 
of its association with altered wall shear stress. ${ }^{9,10}$ The current speckle tracking imaging method in echocardiography has proven to be a valuable diagnostic tool for the segment-wise analysis of rotation and deformation, increasingly used in clinical diagnosis. ${ }^{11}$ Modeling and calculations regarding the aortic wall properties represent a "snapshot" of a current status. Although material parameters and wall motion characteristics give an improved indication for the likelihood of aortic rupture, they remain to be further worked out. We know about a progression of aortic dilation in case of aneurysms of approximately $0.10 \mathrm{~cm} /$ year on average in the ascending aorta. ${ }^{1}$ When the detailed current speckle tracking analysis could be supplemented with additional flow data, the progression analysis will be improved. Although the present operative risk of elective replacement of the ascending aorta is low, we should be attended for the right indication at the right time. Especially, in terms of a more conservative management of aortic dissections considered for long-term survival, computer-assisted wall motion strain measurement with 3D ultrasound speckle tracking and fitted finite element analysis could be helpful. ${ }^{12}$

\section{Conclusion}

3D echocardiographic analysis together with speckle tracking evaluation of wall movement and subsequent finite element analysis opens the way to describe the mechanical behavior of the aortic root and supports clinical decision for intervention or conservative therapy.

\section{Conflict of Interest}

The authors declare that they have no conflict of interest.

\section{References}

1 Elefteriades JA. Indications for aortic replacement. J Thorac Cardiovasc Surg 2010;140(6, Suppl):S5-S9, discussion S45-S51

2 Karatolios K, Wittek A, Nwe TH, et al. Method for aortic wall strain measurement with three-dimensional ultrasound speckle tracking and fitted finite element analysis. Ann Thorac Surg 2013;96(5):1664-1671

3 Sievers HH, Schmidtke C. A classification system for the bicuspid aortic valve from 304 surgical specimens. J Thorac Cardiovasc Surg 2007;133(5):1226-1233

4 Wittek A, Karatolios K, Bihari P, et al. In vivo determination of elastic properties of the human aorta based on 4D ultrasound data. J Mech Behav Biomed Mater 2013;27:167-183

5 Clouse WD, Hallett JW Jr, Schaff HV, et al. Acute aortic dissection: population-based incidence compared with degenerative aortic aneurysm rupture. Mayo Clin Proc 2004;79(2):176-180

6 Davies RR, Goldstein LJ, Coady MA, et al. Yearly rupture or dissection rates for thoracic aortic aneurysms: simple prediction based on size. Ann Thorac Surg 2002;73(1):17-27, discussion 27-28

7 von Spiczak J, Crelier G, Giese D, Kozerke S, Maintz D, Bunck AC. Quantitative analysis of Vortical blood flow in the thoracic aorta using 4D phase contrast MRI. PLoS ONE 2015;10(9):e0139025

8 Bissell MM, Hess AT, Biasiolli L, et al. Aortic dilation in bicuspid aortic valve disease: flow pattern is a major contributor and differs with valve fusion type. Circ Cardiovasc Imaging 2013;6(4):499-507

9 Barker AJ, Markl M, Bürk J, et al. Bicuspid aortic valve is associated with altered wall shear stress in the ascending aorta. Circ Cardiovasc Imaging 2012;5(4):457-466

10 Mahadevia R, Barker AJ, Schnell S, et al. Bicuspid aortic cusp fusion morphology alters aortic three-dimensional outflow patterns, wall shear stress, and expression of aortopathy. Circulation 2014;129(6):673-682

11 Ammar KA, Paterick TE, Khandheria BK, et al. Myocardial mechanics: understanding and applying three-dimensional speckle tracking echocardiography in clinical practice. Echocardiography 2012; 29(7):861-872

12 Di Eusanio M, Berretta P, Cefarelli M, et al. Total arch replacement versus more conservative management in type A acute aortic dissection. Ann Thorac Surg 2015;100(1):88-94 